\title{
DIETARY PROTEIN INTAKE PATTERN AND SOURCES AND THEIR ASSOCIATIONS WITH MUSCLE AND PHYSICAL FUNCTION MEASURES IN OLDER CHINESE ADULTS WITH SARCOPENIA IN HONG KONG
}

\author{
L.-Y. Zhu, R. Chan, L. Li, T. Kwok, J. Woo
}

\begin{abstract}
Background: Protein intake is a major risk factor of sarcopenia. To combat sarcopenia, strategies focused on providing sufficient high quality dietary protein are required. Objectives: We aimed to identify the pattern of dietary protein intake and its association with muscle and physical functions among community-dwelling sarcopenic Chinese older adults in Hong Kong. Design: Baseline data of a randomized controlled trial in sarcopenia were analyzed. Setting: Participants who were ambulant and could travel to the assessment centre at a regional hospital in Hong Kong were recruited in nearby community elderly centers, nursing homes and other institutional settings. Participants: A total of 113 Chinese older adults aged 65 or above who had sarcopenia defined using The Asia Working Group Criteria for Sarcopenia were recruited. Measurements: Dietary data and muscle function tests were measured. Results: Although the energy intake (mean \pm standard deviation: $1491.7 \pm 338.6 \mathrm{kcal} / \mathrm{d}$ in female, $1738.1 \pm 392.9 \mathrm{kcal} / \mathrm{d}$ in male) was lower than the recommended daily energy requirement, protein intake averaged $1.6 \pm 0.5 \mathrm{~g} / \mathrm{kg}$ body weight/day, which was high compared to the current Recommended Daily Allowance (RDA, $0.8 \mathrm{~g} / \mathrm{kg}$ body weight/day for older people). Animal and plant sources contributed to $62 \%$ and $38 \%$ respectively of the total protein intake. Dietary protein intake was not evenly distributed throughout the day. Physical Activity Scale for the Elderly (PASE) score was more predictive of muscle mass and functions compared to protein intake and sources. Conclusions: Our findings showed that PASE was more predictive of muscle mass and functions compared to protein intake and sources, and there was a minimal association between protein intake and muscle performance measures in our community-dwelling sacropenic older adults. The protein replete state of our study population may explain these findings. The observations that an uneven distribution of protein intake throughout a day may suggest the need to increase protein intake at breakfast among Chinese sarcopenic older adults.
\end{abstract}

Key words: Sarcopenia, protein, muscle, Chinese.

\section{Introduction}

Sarcopenia, which is represented by progressive loss of muscle mass and physical function, is a common consequence of aging associated with multiple comorbidities and poor health outcomes. Using the criteria of the Asian working group to define sarcopenia, about $9 \%$ older men and 5\% older women in the community had sarcopenia in Hong Kong. A healthy lifestyle with appropriate diet and physical activity level is important for the prevention of sarcopenia (1).

Epidemiological evidence indicates that having dietary protein intake level higher than the current recommended dietary allowance (RDA, $0.8 \mathrm{~g} / \mathrm{kg}$ body weight/day)

1. Department of Medicine and Therapeutics, The Chinese University of Hong Kong, Rm 124021, 10/F Lui Che Woo Clinical Sciences Building, Prince of Wales Hospital, Shatin Hong Kong SAR

Corresponding Author: Ruth Chan, Department of Medicine and Therapeutics, The Chinese University of Hong Kong, Rm 124021, 10/F Lui Che Woo Clinical Sciences Building, Prince of Wales Hospital, Shatin Hong Kong SAR, ruthchansm@cuhk.edu.hk; Tel.: + 852-3505-2190; Fax: +852-2637-9215 is beneficial for older adults especially for those at risk of muscle loss (2). To maintain and regain lean muscle mass, a total protein intake of 1 to $1.2 \mathrm{~g} / \mathrm{kg}$ body weight per day by older adults has been proposed according to the recent PROT-AGE study group (3). For protein quality, whey protein is preferred as it has a relatively high leucine content which is essential for muscle protein synthesis. Although animal protein is preferred over plant protein in providing leucine, plant protein has also been proven to be beneficial for muscle protein anabolism $(4,5)$. Yet limited studies have directly compared the ability of animal-derived protein with that of the plant protein regarding their distinct chronic influence on muscle mass and muscle function among elderly (6). Uneven distribution of protein intake over a 24-h period other than adequate quantity may also affect muscle mass and functions (7) since the ability to store excess protein for later anabolic use is limited in older adults (8). Thus there may be no benefit for muscle anabolism in elderly if a single large serving of protein is consumed. Instead, an even distribution of a moderate consumption of high 
quality protein of $25-30 \mathrm{~g}$ per meal throughout the day has been proposed (9).

Older adults are prone to be at risk of energy and protein malnutrition due to several causes. For example, chewing problems and loss of appetite are common among elderly and both are linked with decreased food intake in older adults. In addition, taste perception decreases with age. Changes in taste and smell occurring with ageing may influence the food choices and limit the type and the amount of food consumed by the older adults and possibly increase the risk of malnutrition (10). Studies have shown that deficiencies in energy, protein, calcium, vitamin D and iron are common among older adults, and deficiencies in these nutrients, in particular protein were strongly associated with loss of muscle strength and low gait speed and these would ultimately accelerate the progression of sarcopenia (11). However, a framework of dietary nutrient intakes for sarcopenic elderly is still lacking. Data on the pattern of dietary protein intake of sarcopenic elderly are also limited. Furthermore, findings regarding dietary protein intake in terms of sources and their associations with muscle functions and physical performance among sarcopenic elderly have not been well documented yet. As nutrition is one of the key factors to modulate the onset and progression of sarcopenia, more studies of dietary protein intake pattern among sarcopenic elderly would yield valuable information to develop effective strategy to further optimize the muscle protein synthesis and reverse the status of muscle loss. In view of limited data on the dietary protein intake pattern of sarcopenia subjects in the literature, we aimed to examine such pattern and identify its relationship with muscle and physical function measures in a sample of community-dwelling Chinese older adults with sarcopenia in Hong Kong.

\section{Methods}

\section{Study Population}

A total of 113 sarcopenic Chinese older adults living in the Hong Kong community (female $n=87$ ) from a clinical trial (ClinicalTrials.gov Identifier: NCT02374268) assessing the role of exercise and nutrition in sarcopenia were recruited. People aged 65 years and over who were ambulant and could travel to the assessment centre at a regional hospital in Hong Kong were recruited in the regional hospital, nearby community elderly centres, nursing homes and other institutional settings. A brief screening of sarcopenia including handgrip strength and gait speed measurements, as well as cognitive status based on the Chinese version of Mini Mental Status Examination (MMSE, eligible score $\geq 18$ ) (17) was conducted to identify potentially eligible subjects. Potential subjects were further undertaken detailed body composition assessment by dual X-ray absorptiometry (DXA, Hologic QDR-4500W densitometer, Waltham,
Mass, USA) to check for their eligibility. Sarcopenia status was defined by the criteria of Asian Working Group. Sarcopenia was described as low muscle mass $(7.0 \mathrm{~kg}$ / $\mathrm{m}^{2}$ for men and $5.4 \mathrm{~kg} / \mathrm{m}^{2}$ for women by using DXA) plus low muscle strength (grip strength $<26 \mathrm{~kg}$ for men and $<18 \mathrm{~kg}$ for women) and/or low physical performance (usual gait speed $<0.8 \mathrm{~m} / \mathrm{s}$ ) (1). Subjects with MMSE score below 18, self-reported allergy to the ingredients of the nutrition supplement, cancer conditions undergoing treatment, poorly controlled or unstable diabetes, hypertension, chronic obstructive pulmonary disease, cardiovascular disease and unhealed bone fracture were excluded. All participants gave written informed consent. The study was approved by the Clinical Research Ethics Committee of The Chinese University of Hong Kong. Baseline dietary data and data on muscle and physical function measures were used and presented in this paper.

\section{Dietary assessment}

Dietary data were collected by trained research staff using a three-day diet record which included record on two non-consecutive weekdays and one weekend day. Subjects received a brief guideline on how to estimate the food amounts, portion and utensil sizes. The food items and reference portion size (i.e. Chinese bowl) in our dietary records were similar to our previous studies (12). Three-day diet record was completed by subjects before the study visit and checked by trained research staff on the visit day. Cooked dishes and non-commercial processed foods with multiple ingredients were disaggregated into individual food items. Daily energy and protein intakes and consumption of food groups were calculated using the nutrition analysis software Food Processor Nutrition Analysis and Fitness software version 8.0 (ESHA Research, Salem, USA), in which details of the nutrient content of various local foods based on the food composition tables from China and Hong Kong (13) were added.

Protein intake data included amount, food sources and distribution among three main meals and snack. Dietary energy intake and protein intake were compared with those of the sex- and age-specific Chinese dietary reference intakes (version 2013) (14).

\section{Appendicular skeletal muscle mass}

Body composition was measured using DXA (Hologic Discovery A, equipped with software Apex 3.3, Bedford, MA, USA). Calibration with a Hologic body composition spine phantom was performed daily. 


\section{Physical activity pattern, as well as muscle and physical function measurements}

The muscle power of the upper body was tested by seated medicine ball throw test in which subjects were asked to sit in the chair and hold the ball in both hands. They were then instructed to push the ball away from the center of their chest as far as possible. Three practice trials were provided and the average of the test results was recorded (15). Subjects were asked to perform three attempts of their dominant leg extension strength on a fixed chair. The maximum and average of the data were recorded (16). Data on physical activity pattern were assessed using the Physical Activity Scale for the Elderly (PASE). It was a 12-item scale measuring the average time spent in leisure, household and occupational activities for elderly subjects over the previous seven days before the study visit. Higher score indicates higher physical activity level. Gait speed was measured using the best time in seconds to complete a walk along a 6-meter usual straight path.5-chair stands was used to measure the muscle strength of the lower body. 6-minute walk test evaluated the maximum distance that could be covered along an 18-m long corridor during a 6-minute period. Participants were instructed to walk along the walkway as far as they could and to stop when needed. Leg extension was measured by Takei Physical Fitness Test Back Strength dynamometer (T.K.K. 5002 BACK-A). The quality of life was detected by SF-12 including the physical component summary (PCS) and mental component summary (MCS) scores (17).

\section{Statistical analysis}

Data analysis was performed using the statistical package SPSS version 21.0 (SPSS Inc., Illinois, US). Data was checked for normality by descriptive analysis. Mean and standard deviation (SD) or frequency and percentage of subjects' age, body mass index (BMI), energy intake, total protein intake, animal protein intake, plant protein intake, protein intake relative to body weight, the distribution of dietary protein intake throughout the day, as well as the top five protein sources at various meals throughout the day were derived. Pearson's correlation coefficient was used to examine the correlation between protein intake and energy intake. Multivariate linear regression analyses were conducted to examine the association of dietary protein intake and sources with muscle and physical function outcomes with adjustment for age, sex and PASE. All statistical tests were set at 2-sided and $P$ values less than 0.05 were considered statistically significant.
Table 1

Baseline characteristics of the subjects $(n=113)$

\begin{tabular}{|c|c|c|c|}
\hline & Mean \pm SD & $\%$ & $\begin{array}{c}\text { EER / } \\
\text { RNI }\end{array}$ \\
\hline Age (years) & $73.9 \pm 6.9$ & & \\
\hline Female $(\%)$ & & 76.9 & \\
\hline CMMSE score & $27.5 \pm 2.8$ & & \\
\hline Maximum handgrip strength $(\mathrm{kg})$ & $14.9 \pm 5.5$ & & \\
\hline Usual gait speed $(\mathrm{m} / \mathrm{s})$ & $0.88 \pm 0.36$ & & \\
\hline Diabetes mellitus (\%) & & 13.3 & \\
\hline Hypertension (\%) & & 29.2 & \\
\hline Dyslipidemia (\%) & & 20.3 & \\
\hline $\operatorname{BMI}\left(\mathrm{kg} / \mathrm{m}^{2}\right)$ & $18.9 \pm 1.9$ & & \\
\hline \multicolumn{4}{|l|}{ Energy intake (kcal/d) } \\
\hline Total & $1548.4 \pm 365.2$ & & \\
\hline male & $1738.1 \pm 392.9$ & & 2050 \\
\hline female & $1491.7 \pm 339.6$ & & 1700 \\
\hline \multicolumn{4}{|l|}{ Protein intake $(\mathrm{g} / \mathrm{d})$} \\
\hline Total & $71.6 \pm 21.4$ & & \\
\hline male & $84.3 \pm 19.9$ & & 75 \\
\hline female & $67.8 \pm 20.5$ & & 65 \\
\hline \multicolumn{4}{|l|}{ Physical activity (PASE total score) } \\
\hline Total & $89.2 \pm 44.4$ & & \\
\hline male & $95.5 \pm 49.8$ & & \\
\hline female & $87.5 \pm 43.3$ & & \\
\hline Animal protein $(\mathrm{g} / \mathrm{d})$ & $44.5 \pm 19.2$ & & \\
\hline Plant protein $(\mathrm{g} / \mathrm{d})$ & $27.2 \pm 8.6$ & & \\
\hline$\%$ energy from protein & $18.4 \pm 3.1$ & & \\
\hline $\begin{array}{l}\text { Protein intake relative to body }(\mathrm{g} / \mathrm{kg} \text { of } \\
\text { body weight) }\end{array}$ & $1.6 \pm 0.5$ & & \\
\hline $\begin{array}{l}\% \text { of subjects with protein intake }<1.2 \mathrm{~g} / \\
\mathrm{kg} \text { of body weight }\end{array}$ & & 17.7 & \\
\hline
\end{tabular}

\section{Results}

Descriptive characteristics and dietary energy and protein intake data are presented in Table 1. The mean (SD) age of the studied population was $73.9 \pm 6.9$ years old with a range from 65 to 89 years old and there were $76.9 \%$ female subjects. The mean (SD) BMI of the subjects was $18.9 \pm 1.9 \mathrm{~kg} / \mathrm{m}^{2}$ (range from 12.9 to $23.8 \mathrm{~kg} / \mathrm{m}^{2}$ ). Low energy intake was observed in comparison to the Chinese average daily energy requirements for elderly above 70 years old. The PASE score was similar to that of our previously Hong Kong Mr \& Ms Os follow-up study (total=91.3 \pm 43.0 ) (17). Protein intake was in general adequate. Only 20 subjects failed to meet the requirement of $1.2 \mathrm{~g} / \mathrm{kg}$ body weight per day. Animal and plant sources contributed to $62 \%$ and $38 \%$ respectively of the total protein intake. 
Table 2

Distribution of dietary protein intake of the subjects throughout the day $(n=113)$

\begin{tabular}{|c|c|c|c|c|c|c|}
\hline Protein intake & Breakfast & Morning snack* & Lunch & Afternoon snack* & Dinner & Evening snack* \\
\hline \multicolumn{7}{|l|}{ Mean (SD) } \\
\hline Protein intake $(\mathrm{g} / \mathrm{d})$ & $11.9(5.7)$ & $1.9(3.5)$ & $23.2(11.1)$ & $3.6(4.6)$ & $30.4(12.3)$ & $0.6(1.4)$ \\
\hline Animal protein $(\mathrm{g} / \mathrm{d})$ & $6.1(4.6)$ & $0.8(2.1)$ & $14.7(10.1)$ & $1.6(3.6)$ & $21.1(11.4)$ & $0.2(1.1)$ \\
\hline Plant protein $(\mathrm{g} / \mathrm{d})$ & $5.9(3.7)$ & $1.1(1.9)$ & $8.5(3.6)$ & $2.0(2.1)$ & $9.3(3.9)$ & $0.4(0.8)$ \\
\hline Protein ( $\%$ of total protein intake) & $18.0(8.5)$ & --- & $29.8(9.4)$ & --- & $42.7(11.4)$ & --- \\
\hline Animal protein ( $\%$ of protein intake) & $47.0(27.3)$ & --- & $58.5(18.4)$ & --- & $66.1(16.5)$ & --- \\
\hline Plant protein ( $\%$ of protein intake) & $52.9(27.3)$ & --- & $41.5(18.4)$ & --- & $33.9(16.5)$ & --- \\
\hline
\end{tabular}

${ }^{*}$ Data are not normally distributed. However, the mean and SD values instead of the median and interquartile range are presented for easier interpretation.

In Table 2, dietary protein intake presented an uneven pattern throughout the day and skewed to dinner. Significant low protein intake in breakfast was observed. Animal protein was the dominant protein source in three main meals. The dominant protein sources were red and processed meats, refined grains and sea water fishes for lunch and dinner while milk and grains for breakfast (Table 3). There was no association between plant protein intake and muscle related outcome measures. Only total protein intake and animal protein intake showed positive associations with seated medicine ball throw (muscle power of upper limb). Regression analyses showed PASE as a more significant factor to muscle outcome measures compared to protein intake and sources (Table 4).

\section{Discussion}

The amount of dietary protein intake, food sources and their distribution throughout the day are all important to maximize the response of postprandial muscle protein synthesis (9). In this study, dietary protein intake averaged $67.8 \pm 20.5 \mathrm{~g} / \mathrm{d}$ in women and $84.3 \pm 19.9 \mathrm{~g} / \mathrm{d}$ in men, which met the recommended protein requirement for 70 years old Chinese elderly $(75 \mathrm{~g} / \mathrm{d}$ for men over 70 years old and $65 \mathrm{~g} / \mathrm{d}$ for women). The reported values were similar to those reported in a study conducted by Tieland's team in Netherlands $(71.6 \pm 18.8 \mathrm{~g} / \mathrm{d}$ in women and $81.9 \pm 25.2 \mathrm{~g} / \mathrm{d}$ in men among $75-79$ years old community-dwelling subjects) (18). Multiple consensus statements have argued that a relative protein intake beyond the current RDA ( $0.8 \mathrm{~g} / \mathrm{kg}$ body weight/day) or even up to $1.0-1.5 \mathrm{~g} / \mathrm{kg}$ body weight/day is needed for elderly, in particular for those under disease status (19). Though approximately one-third of population over 50 years old failed to meet the RDA requirement for protein, we observed a sufficient protein intake of an average of $1.6 \pm 0.5 \mathrm{~g} / \mathrm{kg}$ body weight/day in our sarcopenic sample. Moreover, a relatively high animal leucine-rich protein intake pattern was observed in our sarcopenic subjects. The 2010 Dietary Guidelines of Americans proposed that although animal based protein provides better quality of protein, plant based protein consumer can also meet the requirement of essential amino acids, such as leucine, for muscle synthesis (20). Similar to the Korean Nutrition Survey (21), data from our Hong Kong Mr Os and Ms Os study also supported the importance of plant protein in preventing sarcopenia. A purely high animal source of dietary protein may be deleterious for muscle health, possibly in promoting an endogenous acid environment (22). It may be that optimal protein intake should be derived from both animal and plant sources. A diet rich in vegetables, fruits as well as snacks-drinks-milk products was also found to be associated with a lower likelihood of sarcopenia (23).

Although we observed the relative protein intake averaged up to $1.6 \pm 0.5 \mathrm{~g} / \mathrm{kg}$ body weight/day and only 20 subjects failed to meet the requirement of $1.2 \mathrm{~g} / \mathrm{kg}$ body weight per day, we found a lower caloric intake in sarcopenic subjects when compared to the intake value of non-sarcopenic elderly reported in our previous study (23). Such caloric restriction may affect the improvements that the protein intake may have on muscle functions in our sarcopenic elderly. Therefore, all these observations possibly suggest that a balanced diet with adequate caloric and protein intake, and rich in alkaline-forming foods, such as vegetables and fruits may be considered as one of the potential nutritional strategies for sarcopenia prevention. However, future questions to be explored include the impact of cultural dietary differences, as well as the optimal ratio of animal protein to plant protein in the preservation of muscle mass and functions.

Our study showed that the three main meals contributed to more than $85 \%$ of daily total protein intake and the protein intake showed an uneven distribution throughout the day. Consistent with the US older population data (24), our sarcopenic older adults showed a skewed protein intake pattern towards the evening meal. Likewise, Mamerow et al. recently completed a 7-d feeding study to measure the changes of 24-h response of muscle protein synthesis between an even and uneven diet. Over the 24 hours, muscle protein synthesis was 25\% higher in the group with an even protein distribution across the three main meals (7). Our findings of a skewed 

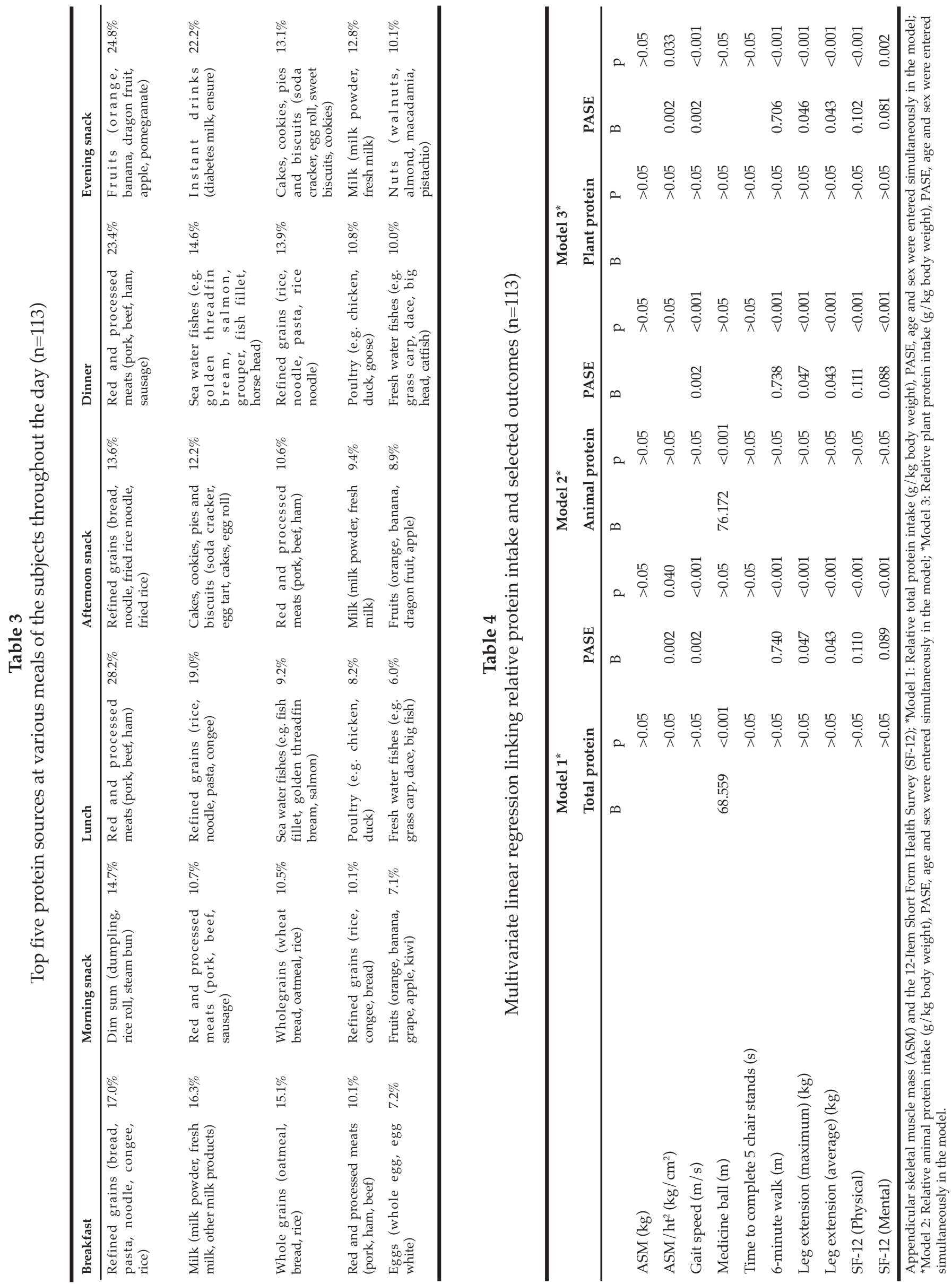
protein intake suggest that there is room for improvement in a more evenly distributed protein meal pattern in our studied population in order to optimize muscle protein synthesis. It has also been shown that ingestion of smaller amounts of dietary protein per meal would attenuate the protein synthetic response in old age, in contrast to young people (8). There would be no advantage of consuming overly large serving of protein in a single meal in older people to preserve muscle mass as the human body has limited ability to store excess protein or amino acid for later use (5). Several studies showed that 25-30 g of dietary protein in each meal is sufficient to maximize protein synthesis among the elderly (9). In our study, we observed an average protein intake of less than 12 $\mathrm{g}$ in breakfast and this intake value was far below the recommended protein intake for a single meal. Therefore, increasing the amount of protein intake in breakfast to at least $25 \mathrm{~g}$ might be a potential dietary strategy to prevent or treat sarcopenia among community-dwelling Chinese older adults in Hong Kong.

It has been proven that combination of exercise and protein ingestion is the most effective way to postpone sarcopenia. Regression analyses showed that PASE was more predictive of muscle mass and functions compared with the amount and source of dietary protein in this population. This may be explained by the protein replete state of our community-dwelling older population, suggesting that protein supplementation would only provide additional benefit to exercise where habitual protein intake is low (24).

Our study has several strengths. First, we used wellrecognized Asia working group definition of sarcopenia to define sarcopenia in the present study and this could allow easier cross-study comparisons in the future. Second, we performed the dietary data analysis using per meal basis instead of daily basis in contrast to previous studies. Our analysis examined detailed dietary patterns and preferences of our sarcopenic elderly. Several limitations should be noted. We used convenience sampling to recruit our sarcopenic subjects from the community and those who joined the study were likely to be more health conscious and have relatively better baseline threshold in terms of health status and nutrient intakes. The sample was also predominately female. Moreover, the aim of the overall trial was to recruit older people to participate in either the intervention arms or the control arm for a 6-month period. Therefore, we intended to recruit relatively healthy older adults because we preferred to have those who could commit to finish the 6-month trial period so as to minimize the dropout rate of the trial. Therefore, our findings may not be generalized to those who are sarcopenic from the hospital settings or the old age homes. Moreover, the present study only captured the recent dietary data using the 3-day diet record. Long-term usual dietary data, such as those assessed using a food frequency questionnaire are not available in the present study. In addition, data on amino acids from diet were not available at the present stage, thus the association of dietary amino acids with sarcopenia remains to be investigated.

In conclusion, there was a minimal association between protein intake and muscle performance measures in our community-dwelling sacropenic older adults. The protein replete state of our study population may explain these findings. The observations that an uneven distribution of protein intake throughout a day may suggest the need to increase protein intake at breakfast among Chinese sarcopenic older adults.

Acknowledgments: This study is supported by grants from the Institute of Ageing, and the Centre for Nutritional Studies of The Chinese University of Hong Kong. The research protocol was approved by ethics committees and that written consent was obtained from all participants.ClinicalTrials.gov Identifier: NCT02374268.

\section{Conflicts of Interest: The authors declare no conflict of interest.}

Ethical standard: The study was conducted according to the German law and to good clinical practice and ethical principles of the Declaration of Helsinki. The Ethics Committee of the Charité - University of Medicine, Berlin, Germany, approved the study.

\section{References}

1. Chen LK, Liu LK, Woo J, et al. (2014) Sarcopenia in Asia: consensus report of the Asian Working Group for Sarcopenia. Journal of the American Medical Directors Association 15:95-101

2. Volpi E, Campbell WW, Dwyer JT, Johnson MA, Jensen GL, Morley JE, Wolfe RR (2013) Is the Optimal Level of Protein Intake for Older Adults Greater Than the Recommended Dietary Allowance? J Gerontol a-Biol 68:677-681

3. Bauer J, Biolo G, Cederholm T, et al. (2013) Evidence-based recommendations for optimal dietary protein intake in older people: a position paper from the PROT-AGE Study Group. Journal of the American Medical Directors Association 14:542-559

4. Luiking YC, Deutz NE, Memelink RG, Verlaan S, Wolfe RR (2014) Postprandial muscle protein synthesis is higher after a high whey protein, leucine-enriched supplement than after a dairy-like product in healthy older people: a randomized controlled trial. Nutrition journal 13:9

5. Arentson-Lantz E, Clairmont S, Paddon-Jones D, Tremblay A, Elango R (2015) Protein: A nutrient in focus. Appl Physiol Nutr Me 40:755-761

6. Haub MD, Wells AM, Tarnopolsky MA, Campbell WW (2002) Effect of protein source on resistive-training-induced changes in body composition and muscle size in older men. The American journal of clinical nutrition 76:511-517

7. Mamerow MM, Mettler JA, English KL, Casperson SL, Arentson-Lantz E, Sheffield-Moore M, Layman DK, Paddon-Jones D (2014) Dietary protein distribution positively influences $24-\mathrm{h}$ muscle protein synthesis in healthy adults. The Journal of nutrition 144:876-880

8. Katsanos CS, Kobayashi H, Sheffield-Moore M, Aarsland A, Wolfe RR (2005) Aging is associated with diminished accretion of muscle proteins after the ingestion of a small bolus of essential amino acids. American Journal of Clinical Nutrition 82:1065-1073

9. Paddon-Jones D, Campbell WW, Jacques PF, Kritchevsky SB, Moore LL, Rodriguez NR, van Loon LJ (2015) Protein and healthy aging. The American journal of clinical nutrition

10. Tieland M, Borgonjen-Van den Berg KJ, Van Loon LJ, de Groot LC (2015) Dietary Protein Intake in Dutch Elderly People: A Focus on Protein Sources. Nutrients 7:9697-9706

11. Kido Y (2015) The Issue of Nutrition in an Aging Society. Journal of nutritional science and vitaminology 61 Suppl:S176-177

12. Chan R, Woo J, Leung J (2011) Effects of food groups and dietary nutrients on bone loss in elderly Chinese population. The journal of nutrition, health \& aging 15:287-294

13. Food Nutrient Finder. Centre for food safety. The Government of the Hong Kong Special administrative region. Web.2017. http://www.cfs.gov.hk / english/nutrient.

14. Society CN. Chinese Dietary Reference intakes. 2013. Science Press, Beijing.

15. Harris C, Wattles AP, DeBeliso M, Sevene-Adams PG, Berning JM, Adams KJ (2011) The Seated Medicine Ball Throw as a Test of Upper Body Power in Older Adults. J Strength Cond Res 25:2344-2348

16. Fragala MS, Alley DE, Shardell MD, et al. (2016) Comparison of Handgrip and Leg Extension Strength in Predicting Slow Gait Speed in Older Adults. Journal of the American Geriatrics Society 64:144-150 
17. Yu R (2014) Incidence, reversibility, risk factors and the protective effect of high body mass index against sarcopenia in community-dwelling older Chinese adults (vol 14, pg 15, 2014). Geriatrics \& gerontology international 14:730-730

18. Tieland M, Borgonjen-Van den Berg KJ, van Loon LJ, de Groot LC (2012) Dietary protein intake in community-dwelling, frail, and institutionalized elderly people: scope for improvement. European journal of nutrition 51:173179

19. Fielding RA, Vellas B, Evans WJ, et al. (2011) Sarcopenia: an undiagnosed condition in older adults. Current consensus definition: prevalence, etiology, and consequences. International working group on sarcopenia. Journal of the American Medical Directors Association 12:249-256

20. United States. Department of Health and Human Services., United States. Department of Agriculture., United States. Dietary Guidelines Advisory Committee. (2010) Dietary guidelines for Americans, 2010. G.P.O., Washington, D.C.
21. Kim J, Lee Y, Kye S, Chung YS, Kim KM (2015) Association of vegetables and fruits consumption with sarcopenia in older adults: the Fourth Korea National Health and Nutrition Examination Survey. Age and ageing 44:96102

22. Chan R, Leung J, Woo J (2015) Association Between Estimated Net Endogenous Acid Production and Subsequent Decline in Muscle Mass Over Four Years in Ambulatory Older Chinese People in Hong Kong: A Prospective Cohort Study. J Gerontol a-Biol 70:905-911

23. Chan R, Leung J, Woo J (2016) A Prospective Cohort Study to Examine the Association Between Dietary Patterns and Sarcopenia in Chinese Community-Dwelling Older People in Hong Kong. Journal of the American Medical Directors Association 17:336-342

24. Breen L, Phillips SM (2013) Interactions between exercise and nutrition to prevent muscle waste during ageing. Brit J Clin Pharmaco 75:708-715 